\title{
A PLASMOID THRUSTER FOR SPACE PROPULSION
}

\author{
Syri J. Koelfgen* and Clark W. Hawk ${ }^{\dagger}$ \\ University of Alabama in Huntsville (UAH) \\ Propulsion Research Center \\ S225 Technology Hall \\ Huntsville, AL 35899 \\ Richard Eskridge, ${ }^{\ddagger}$ James W. Smith ${ }^{\S}$ and Adam K. Martin" \\ NASA Marshall Space Flight Center (MSFC) \\ Propulsion Research Center, TD40 \\ Huntsville, AL 35812
}

\section{ABSTRACT}

There are a number of possible advantages to using accelerated plasmoids for in-space propulsion. A plasmoid is a compact plasma structure with an integral magnetic field. They have been studied extensively in controlled fusion research and are classified according to the relative strength of the poloidal and toroidal magnetic field ( $B_{p}$ and $B_{t}$, respectively). An object with $B_{p} / B_{t}$ " 1 is classified as a Field Reversed Configuration ( $F R C)$; if $B_{p} \approx B_{t}$, it is called a Spheromak. The plasmoid thruster operates by producing FRC-like plasmoids and subsequently ejecting them from the device at a high velocity. The plasmoid is formed inside of a single-turn conical theta-pinch coil. As this process is inductive, there are no electrodes. Similar experiments have yielded plasmoid velocities of at least $50 \mathrm{~km} / \mathrm{s}$, and calculations indicate that velocities in excess of $100 \mathrm{~km} / \mathrm{s}$ should be possible. This concept should be capable of producing Isp's in the range of $5,000-15,000 \mathrm{~s}$ with thrust densities on the order of $10^{5} \mathrm{~N} / \mathrm{m}^{2}$. The current experiment is designed to produce jet powers in the range of $5-10 \mathrm{~kW}$, although the concept should be scalable to several MW's. The plasmoid mass and velocity will be measured with a variety of diagnostics, including internal and external B-dot probes, flux loops, Langmuir

\footnotetext{
* AIAA Student Member, NASA MSFC Graduate Student Researchers Program Fellow \& UAH Graduate Research Assistant

${ }^{\dagger}$ AIAA Fellow, Director of UAH Propulsion Research Center and Professor of Mechanical \& Aerospace

Engineering
}

This paper is declared a work of the U.S. Government and is not subject to copyright protection in the United States. probes, high-speed cameras and a laser interferometer. Also of key importance will be measurements of the efficiency and mass utilization. Simulations of the plasmoid thruster using MOQUI, a time-dependent MHD code, will be carried out concurrently with experimental testing.

\section{INTRODUCTION}

High specific-impulse engines are needed for large $\Delta v$ space missions. ${ }^{1}$ Furthermore, relatively high thrust allows for greater mission flexibility. A plasmoid thruster has many potential advantages for these missions. Since plasmoids are formed inductively in a Field Reversed Configuration (FRC), ${ }^{2}$ they do not suffer from the electrode erosion losses inherent in many advanced propulsion concepts. Due to this electrode-less operation, the device is not limited to specific propellants. As the FRC is a self-contained plasmoid, it is not connected to the walls by magnetic field lines and thus does not require a magnetic nozzle. Consequently, the problem of detachment is lessened or possibly eliminated. Insulation of the plasma from the walls of the device also leads to a higher temperature than that of wall-supported plasmas. In addition, the FRC is relatively free of impurities that would otherwise cool the plasma through line-radiation.

\footnotetext{
‡ AIAA Member, NASA MSFC Aerospace Systems Engineer $\S$ NASA MSFC Mechanical Engineer

"NASA MSFC Physicist
} 
Moreover, this high plasma temperature positively affects efficiency. Plasma resistivity is proportional to $T_{e}^{-3 / 2}$, so a high temperature plasma has a low resistivity $(\eta)$, and therefore a high magnetic Reynolds number, $R=\mu_{0} v L / \eta$, where $\mu_{0}$ is the permeability of free space, $v$ is the plasma velocity, and $L$ is a characteristic scale length. Thus it may be possible to accelerate the plasmoids with high efficiency.

There are, on the other hand, several technological requirements that must be met for successful operation of a plasmoid thruster. Pulsed plasmoid formation requires rep-rates of 10 hertz or greater. $^{3}$ The device would therefore require low-voltage (i.e. less than $20 \mathrm{kV}$ ) formation and acceleration technology such as solid-state switches, or high-voltage (10 - $40 \mathrm{kV})$ switches and high energy density capacitors. Much improvement is required over existing technology for the in-space operation of a plasmoid thruster. The potential advantages however make it a worthy candidate for study. FRC's are empirically well understood, as they have been studied for years as a fusion core. ${ }^{4}$

\section{PLASMOID THRUSTER EXPERIMENT (PTX)}

A plasmoid is an object with an internal magnetic field structure. If the plasmoid has only toroidal current and poloidal magnetic field, it is called a Field Reversed Configuration (FRC); if both the current and magnetic field have poloidal and toroidal components, the plasmoid is classified as a Spheromak (Figure 1). ${ }^{2}$ In practice there is a continuum between the two. The Plasmoid Thruster Experiment (PTX) produces FRC plasmoids in a theta-pinch formation chamber (Figure 2). The plasmoids are produced in a quartz tube situated inside of a theta-pinch coil. The solid aluminum coil has a conical half-angle of $17.5^{\circ}$ and provides a peak magnetic field of 5 kGauss. The quartz tube is connected to a rectangular vacuum chamber for exhausting the plasmoids.

Four cylinders with internal permanent magnets surrounding the vacuum chamber are used to generate a bias field. Before firing, the chamber is filled with gas through the quartz tube. The gas is preionized by a high-voltage DC power supply that is connected between the gas inlet and the chamber (ground). A current pulse is then sent through the theta-pinch coil to produce a reversed magnetic field, i.e. a field in the opposite direction of the bias field. The coil is driven by a $640 \mathrm{nF}, 40 \mathrm{kV}$ capacitor bank which is switched with a Perkin-Elmer spark-gap. The bank is fired by sending an optical trigger signal to a highvoltage pulser that then triggers the spark-gap switch. The capacitors and their associated circuitry sit inside of a tank filled with mineral oil to prevent arcing. When triggered, the spark-gap switch closes, allowing the capacitor bank to discharge through the coil.

In the current experiment, only single FRC's are made. If the concept proves out, the experiment will be upgraded to burst mode operation.

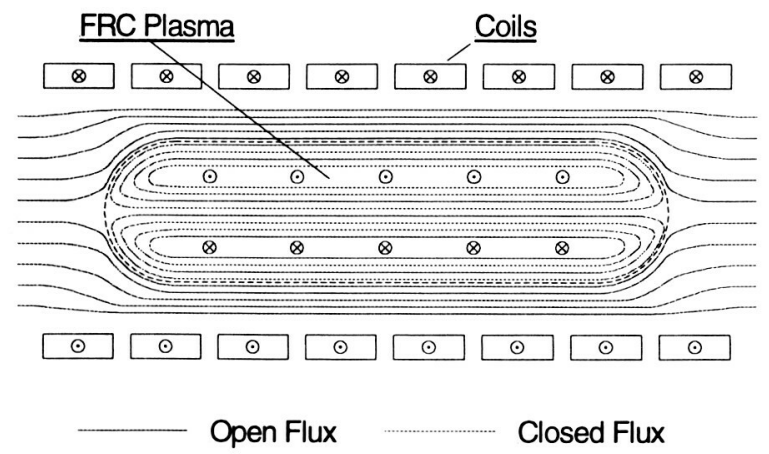

1(a) Field Reversed Configuration (FRC)

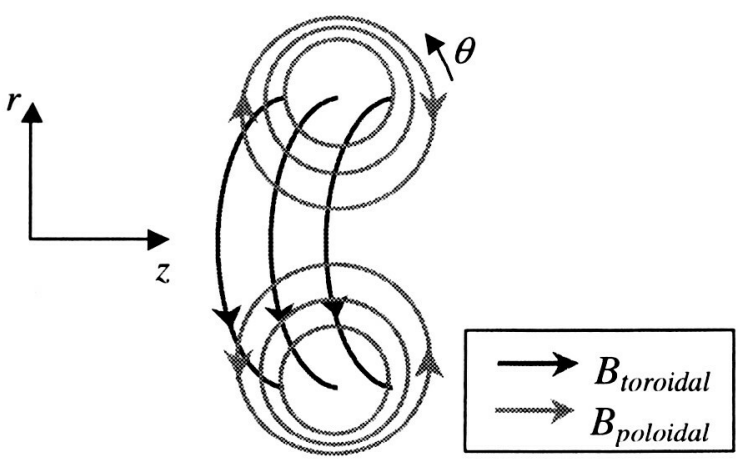

1(b) Spheromak

Figure 1. Plasmoid Magnetic Field Structure

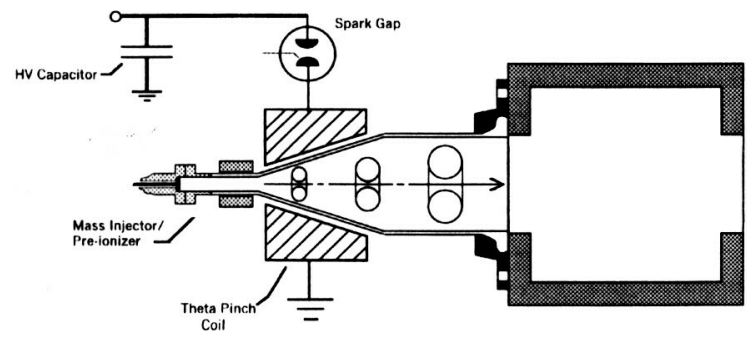

Figure 2. Plasmoid Thruster Experiment (PTX) Schematic 


\section{EXPERIMENTAL OPERATION}

The PTX plasmoids are formed as FRC's in a theta-pinch coil as described previously. Typically, separate capacitor banks are used for preionization, bias-field generation, and reversed field generation (compression). ${ }^{2}$ PTX uses one bank to perform all three stages of formation. This method is referred to as first/second half-cycle formation (Figure 3). This technique was used in the earliest days of FRC research and has the virtue of being relatively simple.

The capacitor bank and coil constitute an L-C circuit, which rings sinusoidally. To begin operation, the gas is first introduced. A static fill is being used at present, but the system is being upgraded to incorporate gas-puff valves. The circuit is then closed with a high-current, highvoltage spark-gap switch, causing the capacitor to discharge through the coil. At time $t_{0}$ the axial magnetic field changes rapidly. This creates an electric field that pre-ionizes the plasma. Simultaneously, the plasma is seeded with the Bfield produced during the first half-cycle. When the current/field swings through zero, the chamber contains a cold, partially ionized plasma with an imbedded magnetic field. As the field increases in the opposite direction, the plasma is shock heated to full-ionization and is compressed.

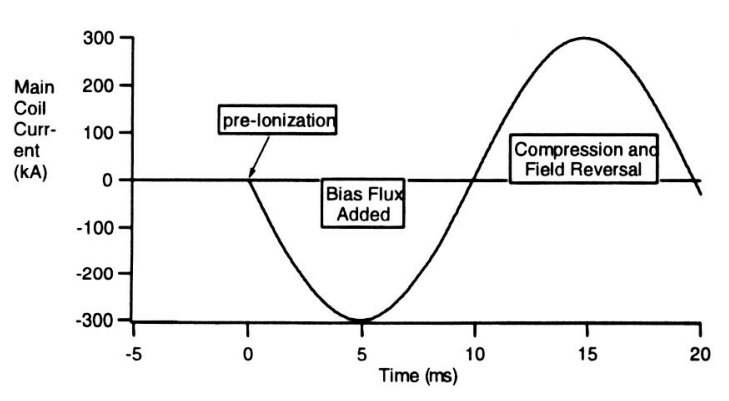

Figure 3. Ideal Coil Current for First/Second Half-Cycle Formation

SPICE was used to simulate the PTX circuit using assumed circuit component values of $640 \mathrm{nF}$ capacitance, $194 \mathrm{nH}$ external inductance, $60 \mathrm{~m} \Omega$ external resistance, $28 \mathrm{nH}$ coil inductance, and $35 \mathrm{kV}$ charging voltage. The peak coil current generated was predicted by SPICE to be $53.4 \mathrm{kA}$ at the quarter-cycle time $t_{1 / 4}=0.6 \mathrm{~ms}$ (Figure 4). When the PTX capacitor bank was actually discharged through the theta-pinch coil, $32 \mathrm{kV}$ of charging voltage was used, resulting in a peak quarter-cycle current of $28 \mathrm{kA}$ (Figure 5), which was lower than predicted by SPICE.

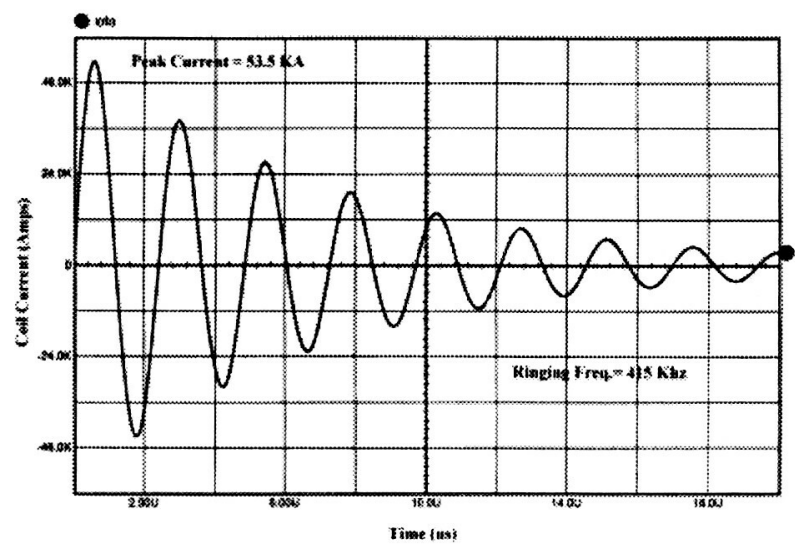

Figure 4. PTX Circuit Simulation using SPICE

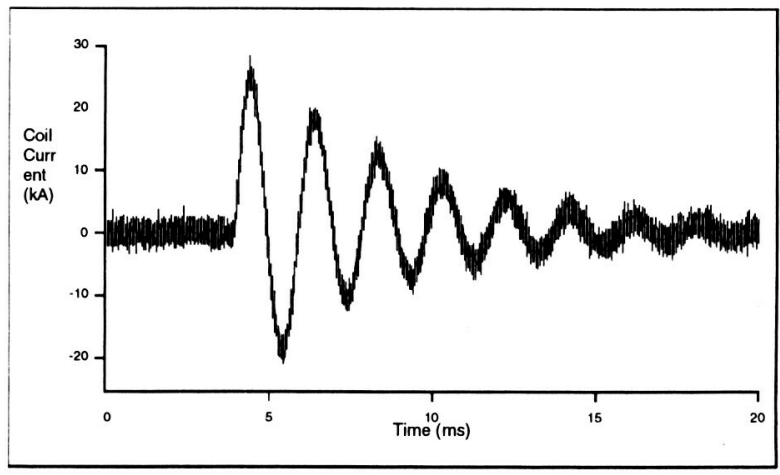

Figure 5. Actual PTX Capacitor Bank Discharge

\section{DIAGNOSTICS}

A variety of diagnostics will be used to study the formation and acceleration of the FRC plasmoids.

Excluded Flux Array

A singular feature of the FRC is that simple external magnetic diagnostics provide detailed information about the internal conditions of the plasma. In equilibrium, the FRC plasmoid sits inside of an open, solenoidal magnetic field; the boundary surface between the open and closed field lines is called the separatrix. FRC's are typically highly prolate, and this is characterized by an elongation $E$, 


$$
E=\frac{l_{\text {sep }}}{2 r_{\text {sep }}}
$$

where $l_{\text {sep }}$ and $r_{\text {sep }}$ are the length and radius of the separatrix respectively. The shape of the separatrix is typically found to be either elliptical, corresponding to a high pressure on the open field lines, or race-track like, corresponding to a low pressure on the open field lines. For a sufficiently prolate FRC, there is a simple formula, the Barnes relation, that links the plasma $\beta$ (the ratio of the plasma pressure to magnetic field pressure) and the separatrix radius by the equation:

$$
\begin{aligned}
& \langle\beta\rangle=\frac{\left\langle n k\left(T_{e}+T_{i}\right)\right\rangle}{\left(\frac{B_{\text {wall }}^{2}}{2 \mu_{0}}\right)} \\
& =1-\frac{1}{2} X_{s}^{2}
\end{aligned}
$$

with

$$
X_{s}=\frac{r_{\text {sep }}}{r_{\text {coil }}}
$$

where $n$ is the plasma density, $k$ is Boltzmann's constant, $T_{e}$ and $T_{i}$ are the electron and ion plasma temperatures respectively, $B_{\text {wall }}$ is the magnetic field at the vacuum wall and $r_{\text {coil }}$ is the radius of the theta-pinch coil.

The separatrix radius is measured using an excluded flux array, which consists of a fluxloop ${ }^{5}$ and associated B-dot probe ${ }^{5,6}$ located beneath every coil, just outside of the vacuum wall (the quartz tube). (Figure 6) The B-dot probe measures $B_{\text {wall }}$ and the flux loop measures the flux within that loop, where flux is given by

$$
\Phi=\pi\left(r_{\text {wall }}^{2}-r_{\text {sep }}^{2}\right) B_{\text {wall }}
$$

The flux within the separatrix is zero by definition. One then defines an 'excluded flux', $\Delta \Phi$, from:

$$
\begin{aligned}
& \Delta \Phi=\pi r_{\text {wall }}^{2} B_{\text {wall }}-\Phi \\
& =\pi r_{\text {wall }}^{2} B_{\text {wall }}-\pi\left(r_{\text {wall }}^{2}-r_{\text {sep }}^{2}\right) B_{\text {wall }} \\
& =\pi r_{\text {sep }}^{2} B_{\text {wall }}
\end{aligned}
$$

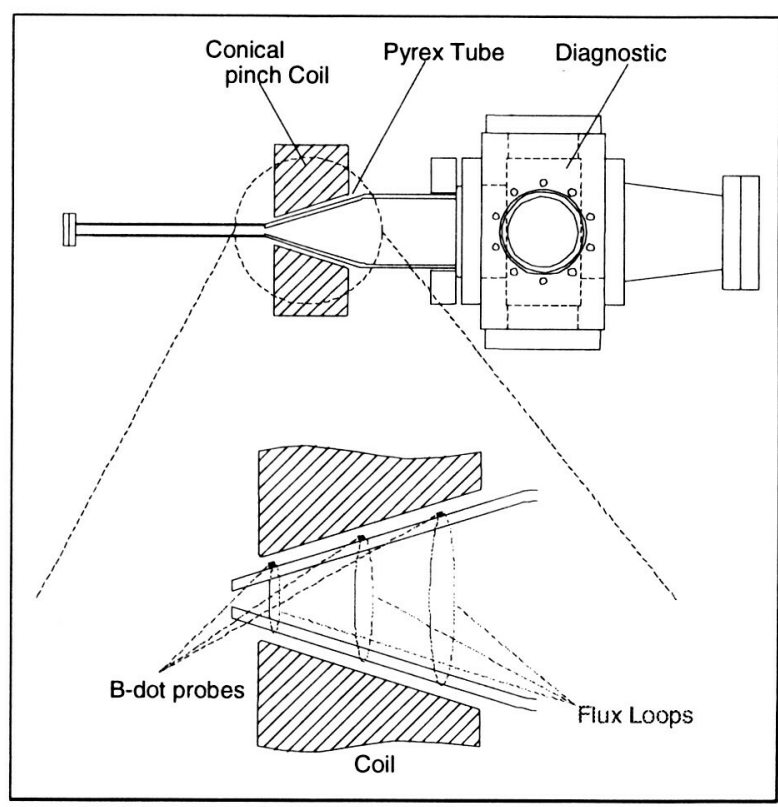

Figure 6. Excluded Flux Array

so that:

$$
r_{\text {sep }}=\sqrt{\frac{\pi r_{\text {wall }}^{2} B_{\text {wall }}-\Phi}{\pi B_{\text {wall }}}}
$$

where $r_{\text {wall }}$ is the radius to the vacuum wall. Hence, from the two independent measurements of $B_{\text {wall }}$ and $\Phi, r_{\text {sep }}$ can be measured at every axial probe/flux-loop location, giving an axial profile of $r_{s e p}$, and therefore the shape of the separatrix, as well as the internal plasma pressure from the Barnes relation.

\section{Internal B-dot Probes}

Internal B-dot probes ${ }^{5,6}$ will be used to measure the magnetic fields in the open flux region, in the exhaust region, and within the FRC itself. Six sets of two B-dot probes (for a total of twelve probes) have been constructed for measuring $B_{z}$ and $B_{\theta}$, the axial and azimuthal magnetic fields, respectively, of the translating plasmoid. (Figure 7) The probes were wound with 36 gauge magnet wire around a $3.15 \mathrm{~mm}$ square form made from G-10 circuit board material. During construction of the probes, the fragile wire was kept taut by winding it around spools mounted to an optics table. 


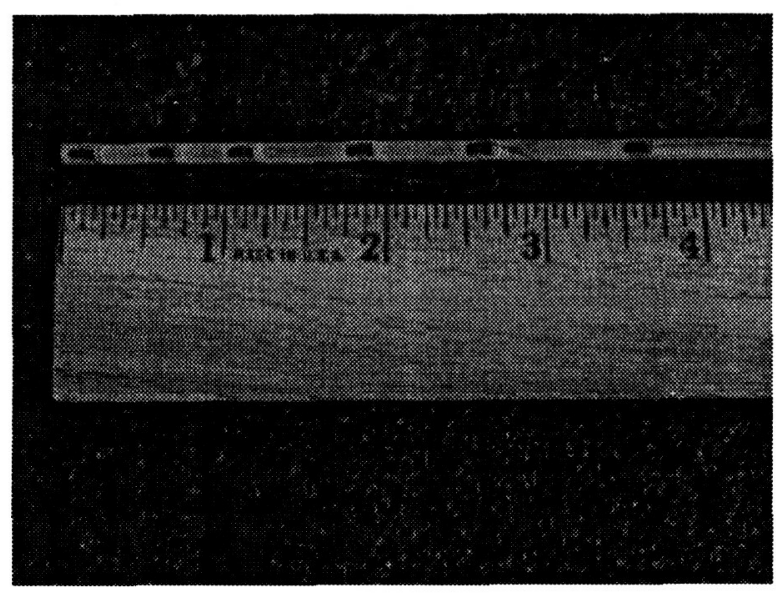

Figure 7. Internal B-dot Probes

A Helmholtz coil (Figure 8) was built for the purpose of calibrating the B-dot probes. The Helmholtz coil is a set of two coaxial current loops with equal radii, separated from each other by that radius. The radius of the PTX Helmholtz coil loops is 2.5 inches.

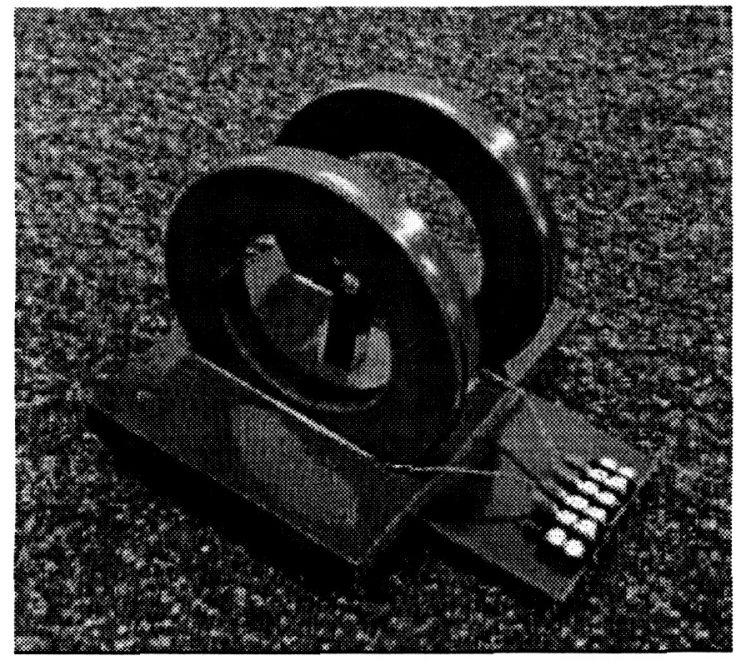

Figure 8. Helmholtz Coil

Equal currents flow in the same direction in each of the current loops. The magnetic field produced by the Helmholtz coil is extraordinarily uniform inside the volume of the coils, hence it provides a valuable calibration device. The Helmholtz coil magnetic field was calibrated with a power supply (current source), multimeter and Hall probe. The magnetic field inside the coils along the " $z$ " axis is shown in figure 9. The measured data is not completely flat in the area of the uniform field, probably due to the fact that the coils were heating up during calibration and changing the effective current. Figure 10 shows the calibration data for the Helmholtz coil.

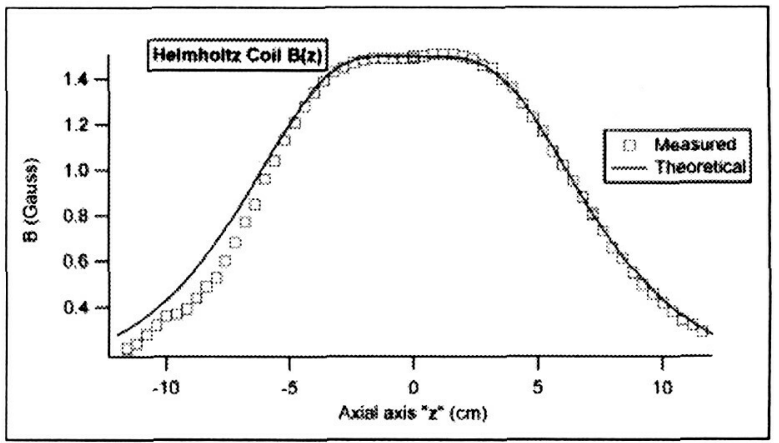

Figure 9. Axial Magnetic Field of Helmholtz Coil

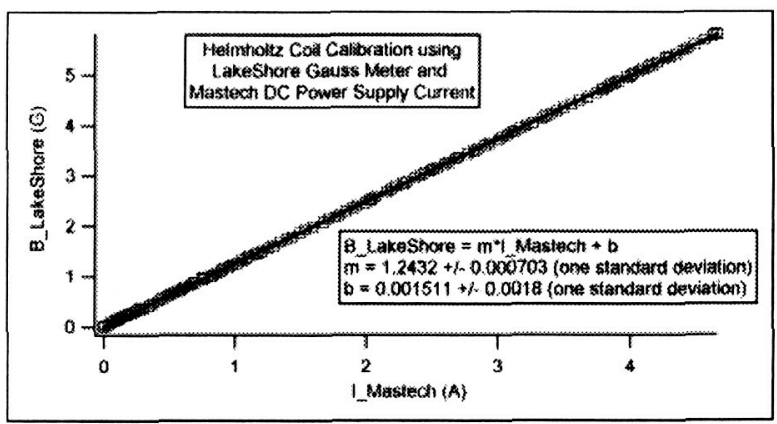

Figure 10. Helmholtz Coil Calibration Curve

In order to calibrate the B-dot probes, the Helmholtz coil was pulsed with a 300 VDC trigger, and the resulting voltage (translated into current and then magnetic field) responses of the Helmholtz coil and B-dot probes were measured with a digital oscilloscope. The magnetic field $B$ seen by the B-dot probe is obtained through the relation

$$
B=\int \dot{B} d t=\frac{1}{N A} \int V d t
$$

where $\dot{B}$ (B-dot) is the change in magnetic field with time, i.e. $\frac{\partial B}{\partial t}$, as measured by the probe, $d t$ is the time of measurement, $N$ is the number of turns of the probe, $A$ is the cross-sectional area of 
the probe, and $V$ is the voltage measured across the leads of the probe.

The calibrated item, $N A$, was found to be within $~ 80 \%$ of the predicted value of $N A=$ $10^{\star}(3.15 \mathrm{~mm})^{2}=9.92 \mathrm{E}-5 \mathrm{~m}^{2}$ for the probes. The frequency response of the B-dot probes was also assessed by applying a sine wave to the Helmholtz coil at frequencies from $1 \mathrm{kHz}$ to $3 \mathrm{MHz}$. Responses of the Helmholtz Coil and the B-dot probes were measured with the oscilloscope. Figure 11 shows a typical plot of the calibration constant $N A$.

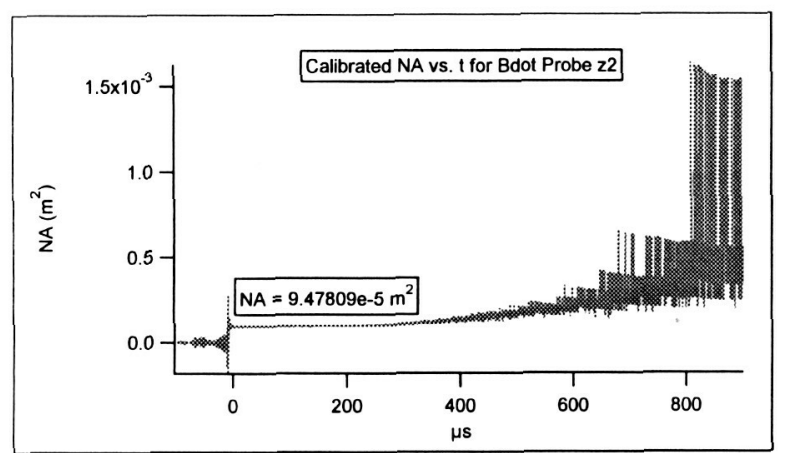

Figure 11. Typical B-dot Probe Calibration Plot

\section{Langmuir Probes}

Quadruple Langmuir probes ${ }^{7}$ will be used to make local measurements of electron temperature $T_{e}$ and number density $n_{e}$ in the open flux region and in the exhaust region. Three of the four probes on the quadruple probe sample the Current-Voltage characteristic, and from this yield measurements of $T_{e}$ and $n_{e}$. The fourth bent probe provides an estimate plasma velocity.

\section{Interferometry}

A heterodyne, quadrature $\mathrm{He}-\mathrm{Ne}$ laserinterferometer will be used to measure the plasma number density in the FRC. This instrument is described briefly in Reference 8 .

In laser interferometry, the laser beam is split into two beams of approximately equal intensity. One, called the scene-beam passes through the plasma; the other, called the reference beam, passes outside of the vacuum chamber. The scene beam acquires a phase-shift due to the plasma given by

$$
\Delta \varphi=\frac{e^{2} \lambda}{4 \pi \varepsilon_{0} m_{e} c^{2}} \int n d l
$$

where $e$ and $m_{e}$ are the charge and mass of the electron respectively, $\varepsilon_{0}$ is the permittivity of free space, $c$ is the speed of light, $\lambda$ is the wavelength of the laser light, and $n$ is the plasma number density (electron number density). The phase shift is proportional to the line-integral of the plasma density along the scene beam.

The reference beam is frequency-shifted by $40 \mathrm{Mhz}$ using an acousto-optic Bragg cell. The scene and reference beams are recombined at a photo-detector and the resulting voltage signal is AC-coupled to an analyzer-circuit. There, the signal passes through a filter that selects the difference frequency. The interference signal (phase shift) is encoded as a frequency modulation of the $40 \mathrm{Mhz}$ carrier signal. This 'heterodyning' preserves the sense of the phase shift when it goes through zero, and makes the instrument relatively insensitive to electrical noise and variations in amplitude.

The analyzer-circuit splits the interference signal into two signals. One of these signals is mixed with the original $40 \mathrm{Mhz}$ carrier signal, and the other is mixed with the carrier signal shifted by $90^{\circ}$. These are then low-pass filtered, yielding two signals proportional to $\sin (\Delta \phi)$ and $\cos (\Delta \phi)$ respectively. From these, $\Delta \phi$ may be reconstructed unambiguously. The line-averaged density is then calculated from this phase-shift. At least 3 , and perhaps as many as 6 chords of interferometry will be available for PTX.

\section{High-speed Photography}

High-speed photography will be used to image the FRC end-on, and to measure its velocity as it is translated. A variety of cameras with nanosecond resolution are available for the high-speed photography. PTX has access to a Cordin 220B camera that can take 8 pictures at 100 million frames/second, a Hamamatsu streakcamera and two Princeton Instruments PI-Max cameras, which have an intensified $C C D$ array ( $512 \times 512$ pixels) and shutter-times down to 2 ns.

\section{Bolometry}

A silicon XUV photo-diode bolometer ${ }^{6}$ will be used to measure the integrated radiation flux from the plasma, which will be instrumental in determining the overall energy balance. 


\section{EXPERIMENTAL OBJECTIVES}

In order to have a high efficiency in a working device, it will be necessary to efficiently ionize the plasma and form the FRC. As with all electric propulsion systems, ionization represents a large frozen-flow loss in a plasmoid thruster. ${ }^{1,2}$ it is necessary to reduce its overall influence on the system energy budget. With the theta-pinch formation method, the plasma is formed in a partially ionized state and is fully ionized during the shock heating caused by the field reversal. It has been found though that FRC equilibrium is sensitive to the pre-ionization. ${ }^{5}$ Various preionization techniques for PTX will be investigated, including ringing magnetic field ionization, coaxial plasma injectors, and perhaps rotating magnetic fields (RMF).

For a practical system, it will also be necessary to minimize resistive losses in the coils (by, for example, liquid nitrogen cooling), and to maximize the recapture of inductive energy in the coils and transmission line.

To evaluate the performance of the plasmoid thruster, measurements used to assess specific impulse, thrust and efficiency will be taken. Since propulsion performance is also characterized by how much of the plasma exits from the device, detachment of the plasmoid from the magnetic field lines will also be investigated. Similar experiments have yielded plasmoid velocities of at least $50 \mathrm{~km} / \mathrm{s}$, and calculations indicate that velocities in excess of $100 \mathrm{~km} / \mathrm{s}$ should be possible. The concept should be capable of producing Isp's in the range of 5,000 15,000 seconds with thrust densities on the order of $10^{5} \mathrm{~N} / \mathrm{m}^{2}$. The current experiment is designed to produce jet powers in the range of $5-10 \mathrm{~kW}$, although the concept should be scalable to several MW's.

Isp-related measurements to be obtained include velocity and mass utilization. Velocity will be measured by use of high-speed photography, the internal B-dot probe array, laser interferometry and the quadruple Langmuir probe. Mass utilization will be evaluated by taking an inventory of the propellant mass put into the device compared to the mass of the plasma coming out. The mass of the plasma emerging from the device will be determined by measuring the density and volume of the ejected plasmoid. Density will be measured using the quadruple Langmuir probe and the laser interferometer. The Langmuir probe will provide a local measurement of electron number density, while the interferometer will make a non-intrusive measurement of line-averaged plasma density. The volume of the plasmoid will be determined by using the internal B-dot probe array as well as the high-speed photography.

Thrust-related measurements to be obtained include plasmoid mass and velocity. The mass and velocity will be acquired as previously described. Overall jet power, and therefore also thrust, will be limited by the rate at which the system can be fired. Currently, the experiment is being fired in single-shot mode, however upgrades to repetitive operation are being developed. Gaspuff valves operable at frequencies of up to $500 \mathrm{hz}$ are being installed. For pulsed operation, it is also necessary to design capacitor banks and switching circuits that can be repetitively fired. This will be done after initial experiments are performed.

One of the advantages of the FRC thruster is that it should behave almost like a mass-driver, ejecting a magnetized plasmoid that is not magnetically attached to the nozzle. It will be necessary however to actually demonstrate this experimentally. Detachment of the plasmoid from the magnetic field lines will be investigated by characterizing how much of the plasmoid plasma stays attached to the field lines with increasing distance from the theta-pinch coil. Magnetic diagnostics and high-speed photography will be used to evaluate the shape and magnetic field structure of the translating plasmoid. In addition to the internal B-dot probe array, an excluded flux array will also be used to this means. It may also be favorable to allow the plasmoid to decay just as it reaches the nozzle (by making $\tau_{\text {flux decay }}$ comparable to $\left.\tau_{\text {transit }}\right)$, so as to recoup, at least partially, the frozen-in magnetic and plasma energy, and convert it into directed kinetic energy in the exhaust.

Simulations of the plasmoid thruster using MOQUI, a time-dependent MHD code, will be carried out concurrently with experimental testing.

\section{INITIAL RESULTS}

Images of PTX translating plasmoids have been captured with the Cordin camera. Figure 12 shows a plasmoid propagating in air at $43 \mathrm{mTorr}$ backfill pressure. The Cordin camera has $8 \mathrm{CCD}$ cameras in a circular configuration that take photos at a rate of 100 million frames/second, with a gate time of $10 \mathrm{~ns}$ per frame. The plasmoid velocity has been estimated from the Cordin images by assessing the amount of time for the plasmoid to traverse the vacuum chamber viewport over the 8 frames. The plasmoid shown 
in Figure 12 (which was frame 6 of the 8) was estimated to have a velocity of $5.3 \mathrm{~km} / \mathrm{s}$.

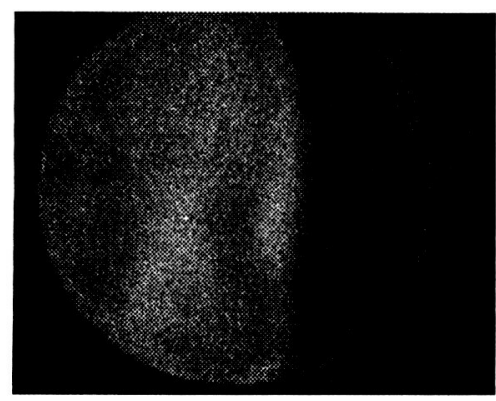

Figure 12. Translating Plasmoid

In order to begin evaluating PTX ionization, the Cordin camera has also been placed at the end of the vacuum chamber so that it is looking into the throat of the theta-pinch coil. For the $6 \% \mathrm{H}_{2}$ in He gas, a 128 mTorr static fill and preionization, a plot of plasmoid images (with a camera shutter speed of $20 \mathrm{~ns}$ ) correlated with the coil current trace was made (Figure 13). From this plot it is proposed that two separate plasmoids are formed during a test firing.

\section{CONCLUSION}

The plasmoid thruster is a promising concept for in-space propulsion with a number of possible advantages. The Plasmoid Thruster Experiment seeks to evaluate the concept. The plasmoid thruster operates by producing FRC-like plasmoids in a theta-pinch coil and subsequently ejecting them from the device at a high velocity. To evaluate the performance of PTX, the plasmoid mass, mass utilization, velocity and thruster efficiency will be measured with a variety of diagnostics, including internal and external B-dot probes, flux loops, Langmuir probes, high-speed cameras and a laser interferometer.

High Speed Plasmoid Images (20 ns Shutter Speed) Correlated with Theta-Pinch Coil Current Data

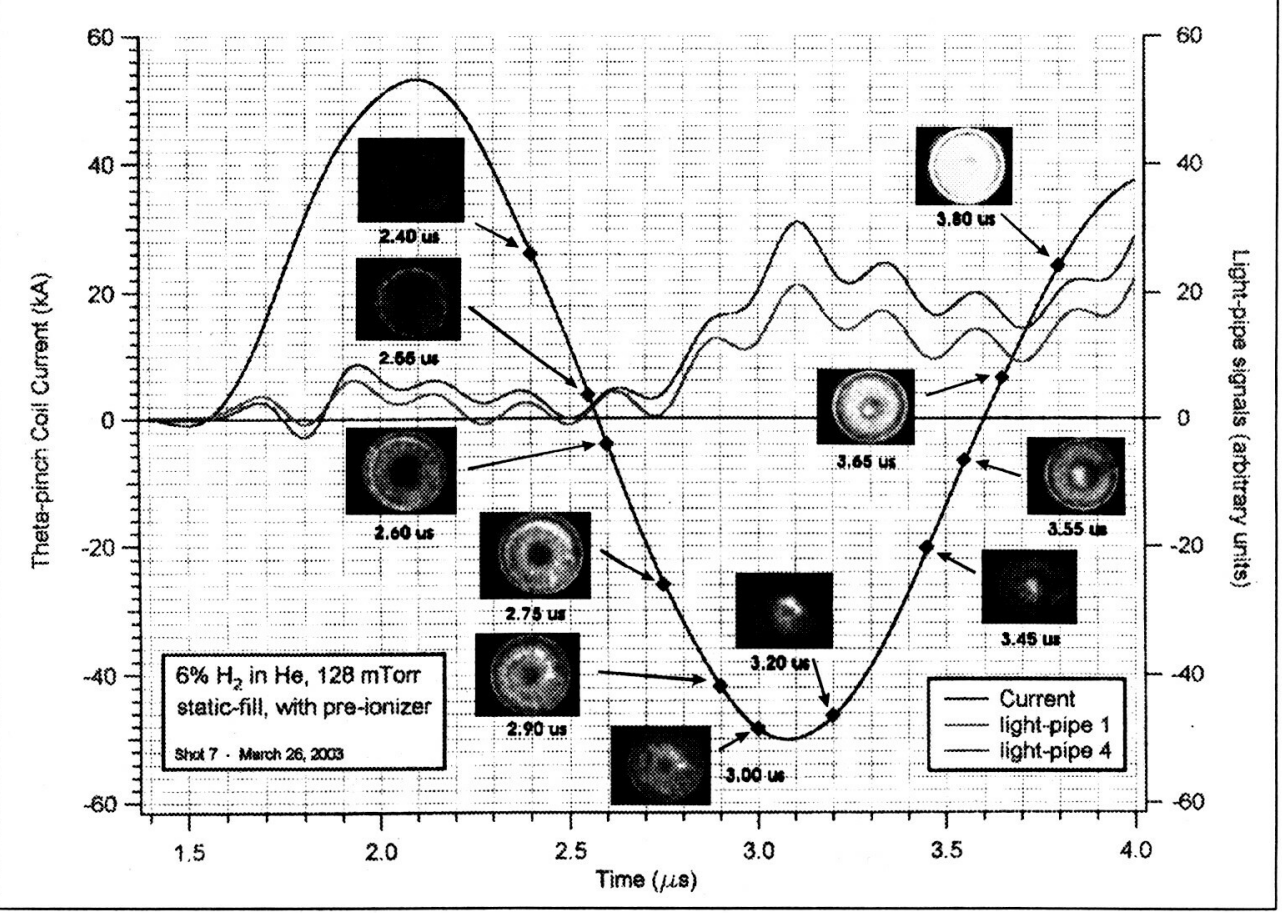

Figure 13. Plasmoid Images with Coil Current 


\section{REFERENCES}

1.) "Physics of Electric Propulsion," Robert G. Jahn, McGraw-Hill, 1968.

2.) "Field Reversed Configurations - Review Paper," M. Tuszewski, Nuclear Fusion 28(11), 1988.

3.) "Pulsed High-Density Fusion Rocket," J. Slough, NASA Fusion Propulsion Workshop, Nov. 8-10, 2000, NASA Marshall Space Flight Center, Huntsville, Alabama.

4.) "Inductive Field-Reversed Configuration Accelerator for Tokamak Fueling," A.L. Hoffman et. al., Fusion Technology, 36, Sep. 1999.

5.) "Principles of Plasma Diagnostics," I. H. Hutchinson, Cambridge University Press, 1987.

6.) "Plasma Diagnostic Techniques," Richard $\mathrm{H}$. Huddlestone and Stanley L. Leonard, Academic Press, 1965.

7.) "Application of a Quadruple Probe Technique to MPD Thruster Plume Measurements," R. L. Burton and S. Del Medico, Journal of Propulsion and Power 9(5), pp. 771-777, Sep. - Oct. 1995.

8.) "Photographic, magnetic, and interferometric measurements of current sheet canting in a pulsed plasma accelerator" T.E. Markusic, E.Y. Choueiri, $37^{\text {th }} \quad$ AIAA/ASME/SAE/ASEE Joint Propulsion Conference \& Exhibit, July 811, 2001, Salt Lake City, Utah, AIAA Paper 2001-3896. 

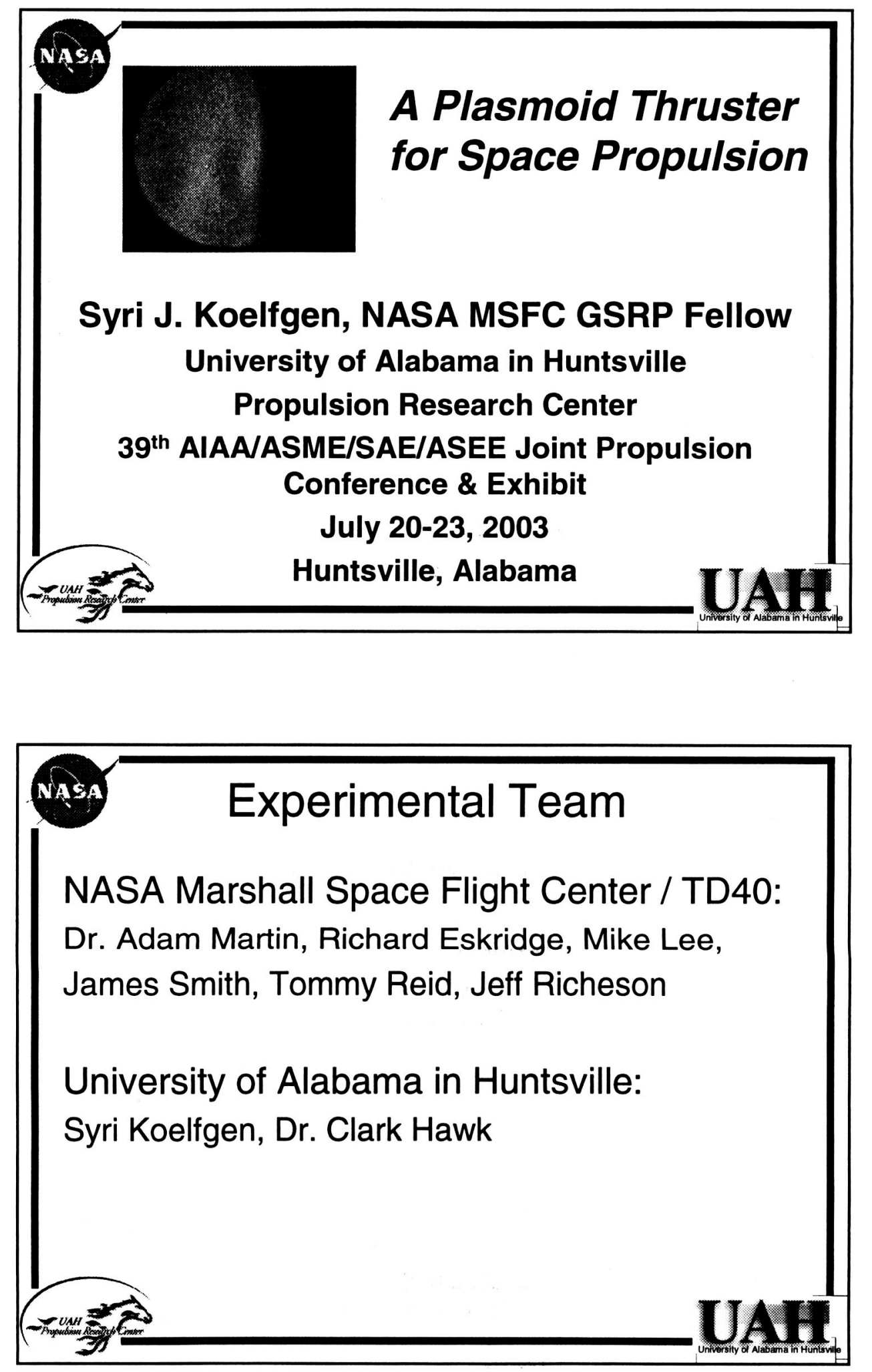


\section{Presentation Outline}

I. Background

II. Plasmoid Propulsion

III. Plasmoid Thruster Experiment (PTX)

IV. Diagnostics

V. Imaging Results

VI. Future Work
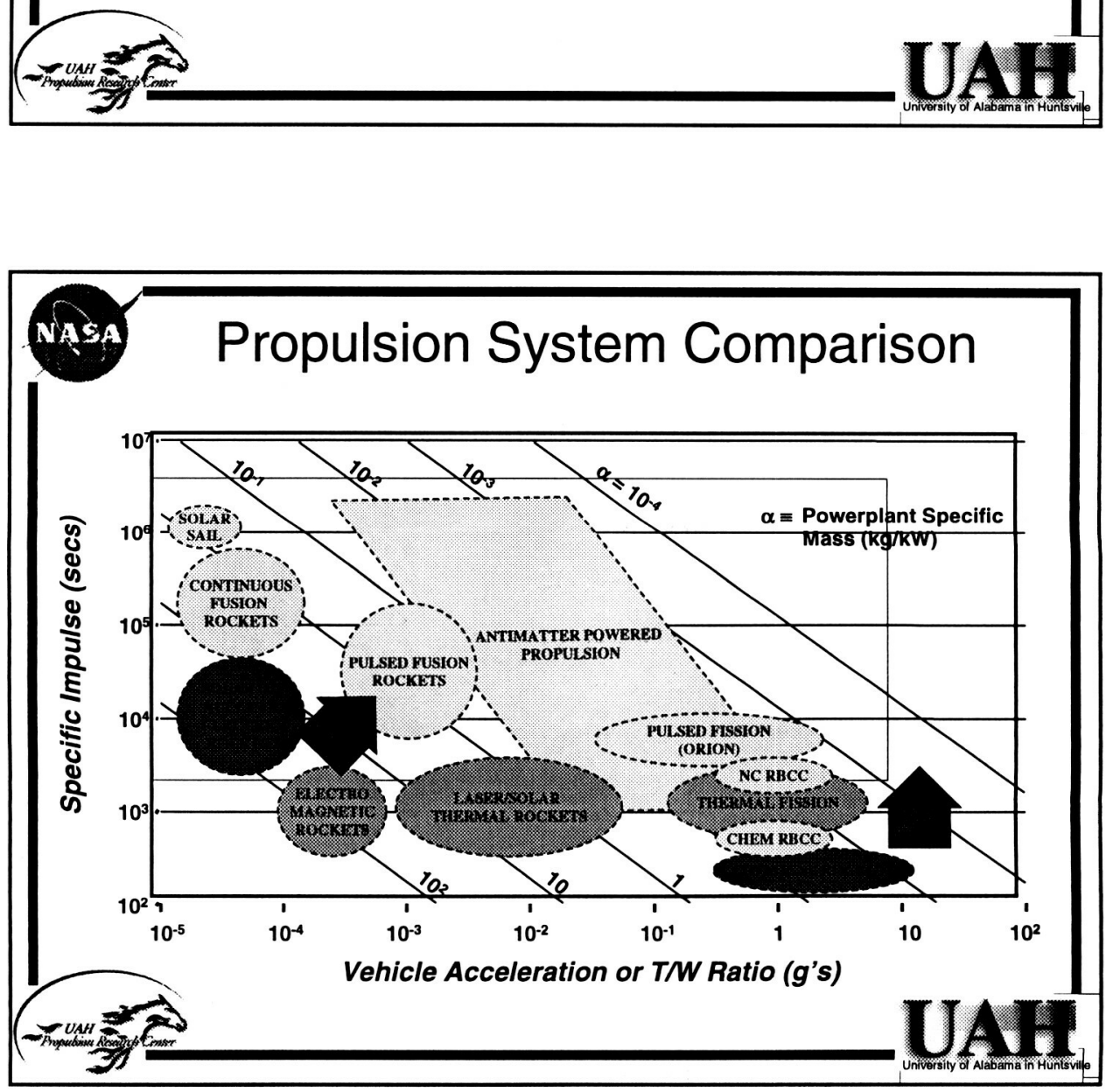

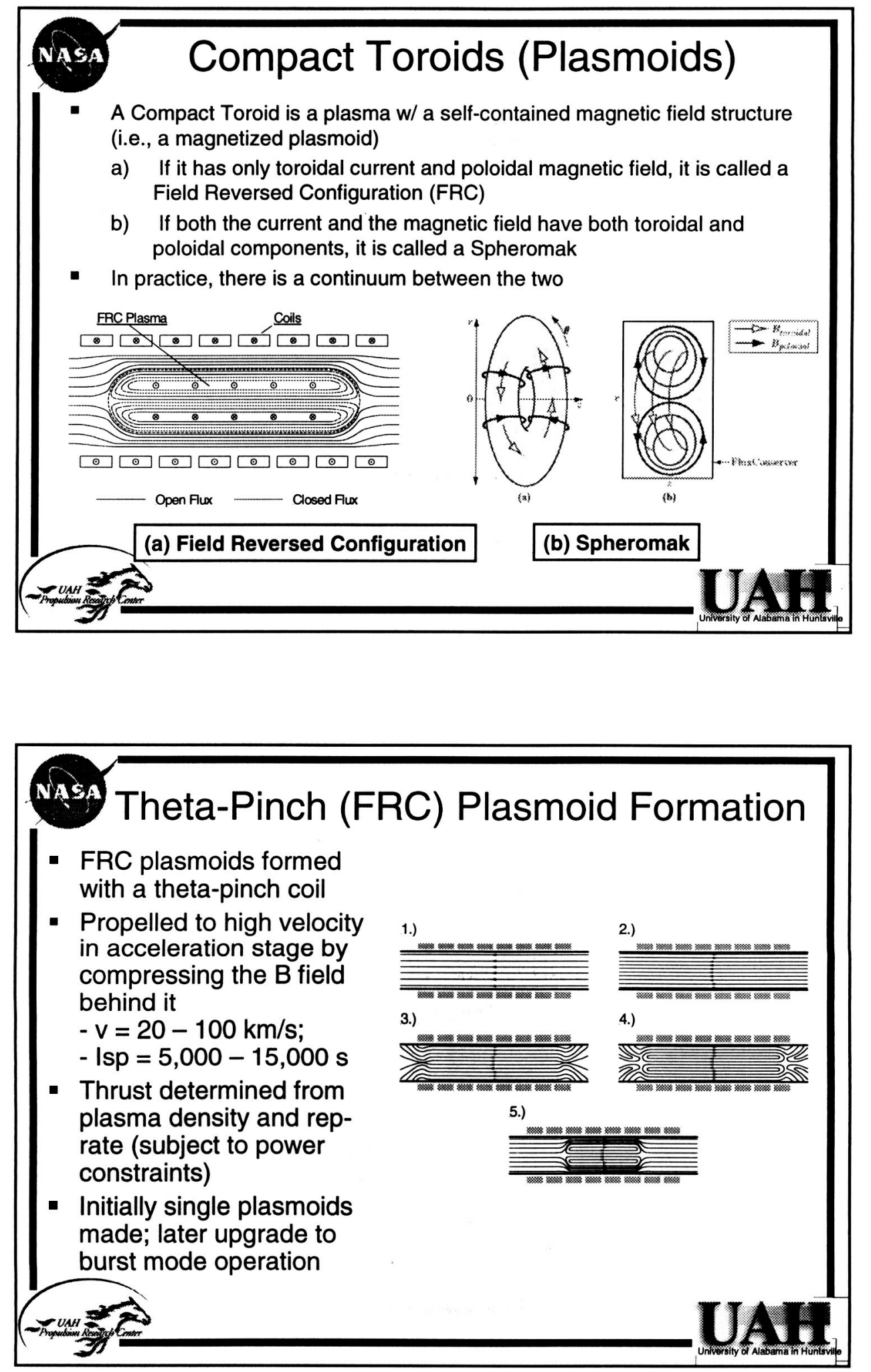


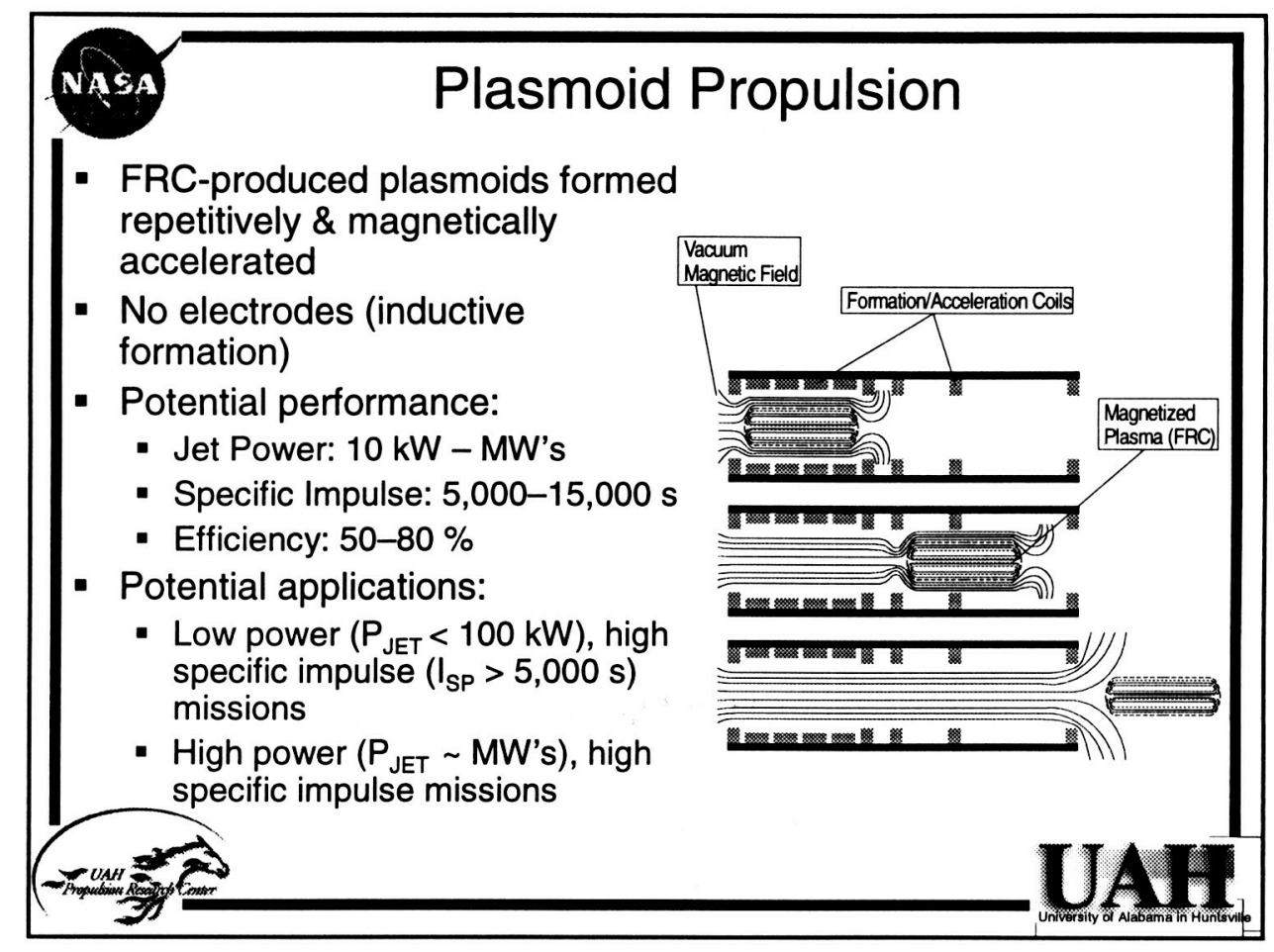

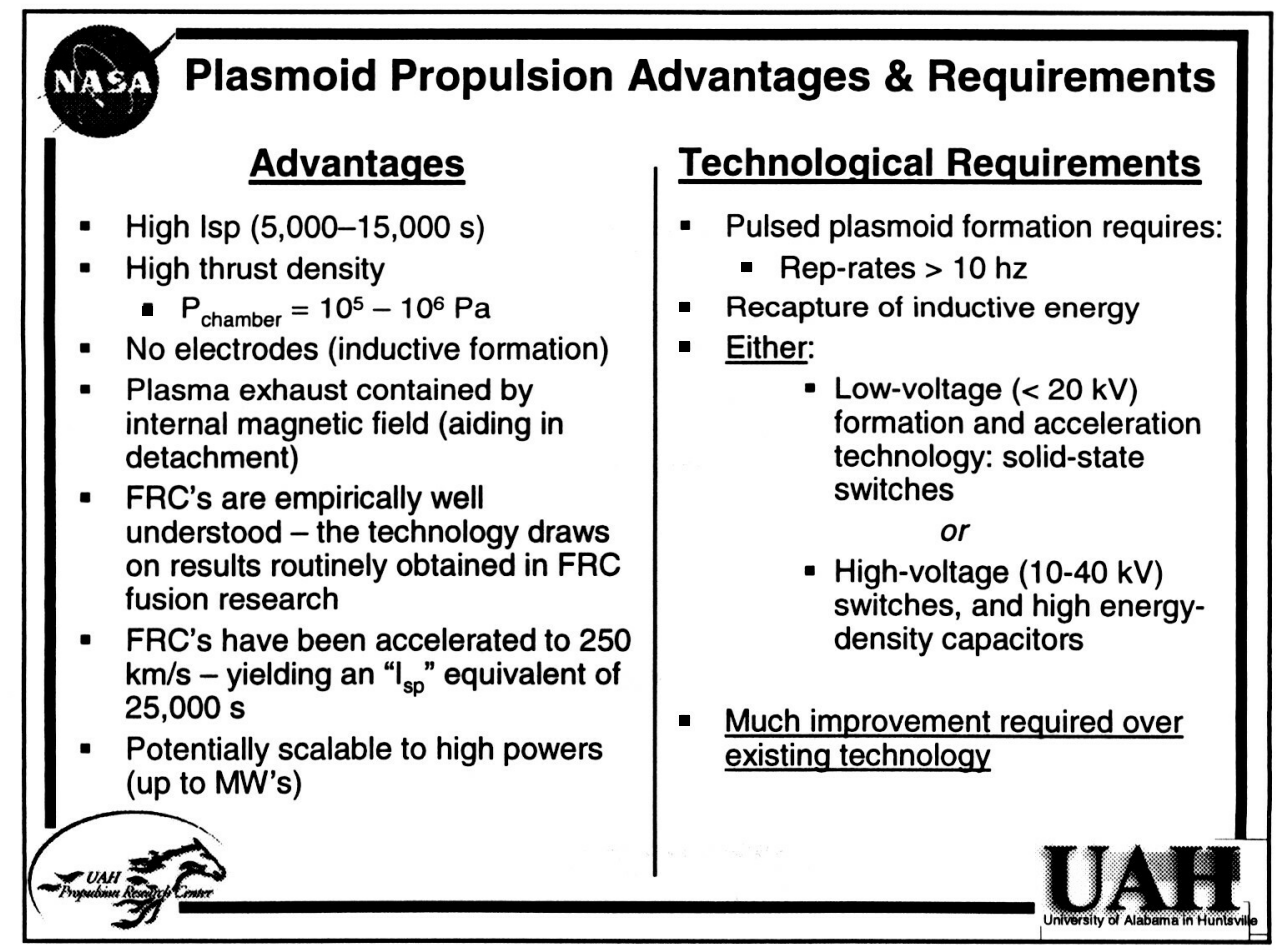



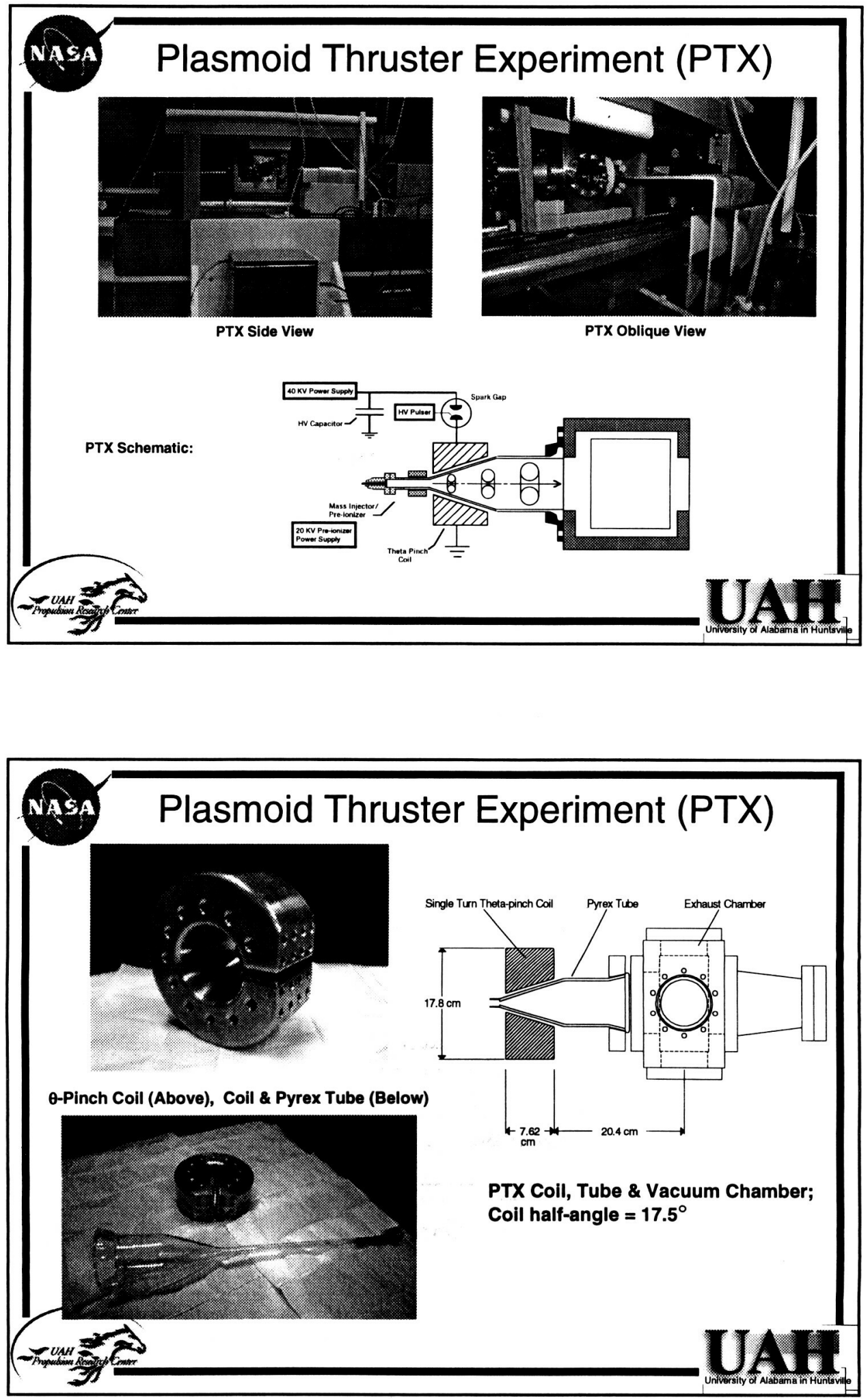

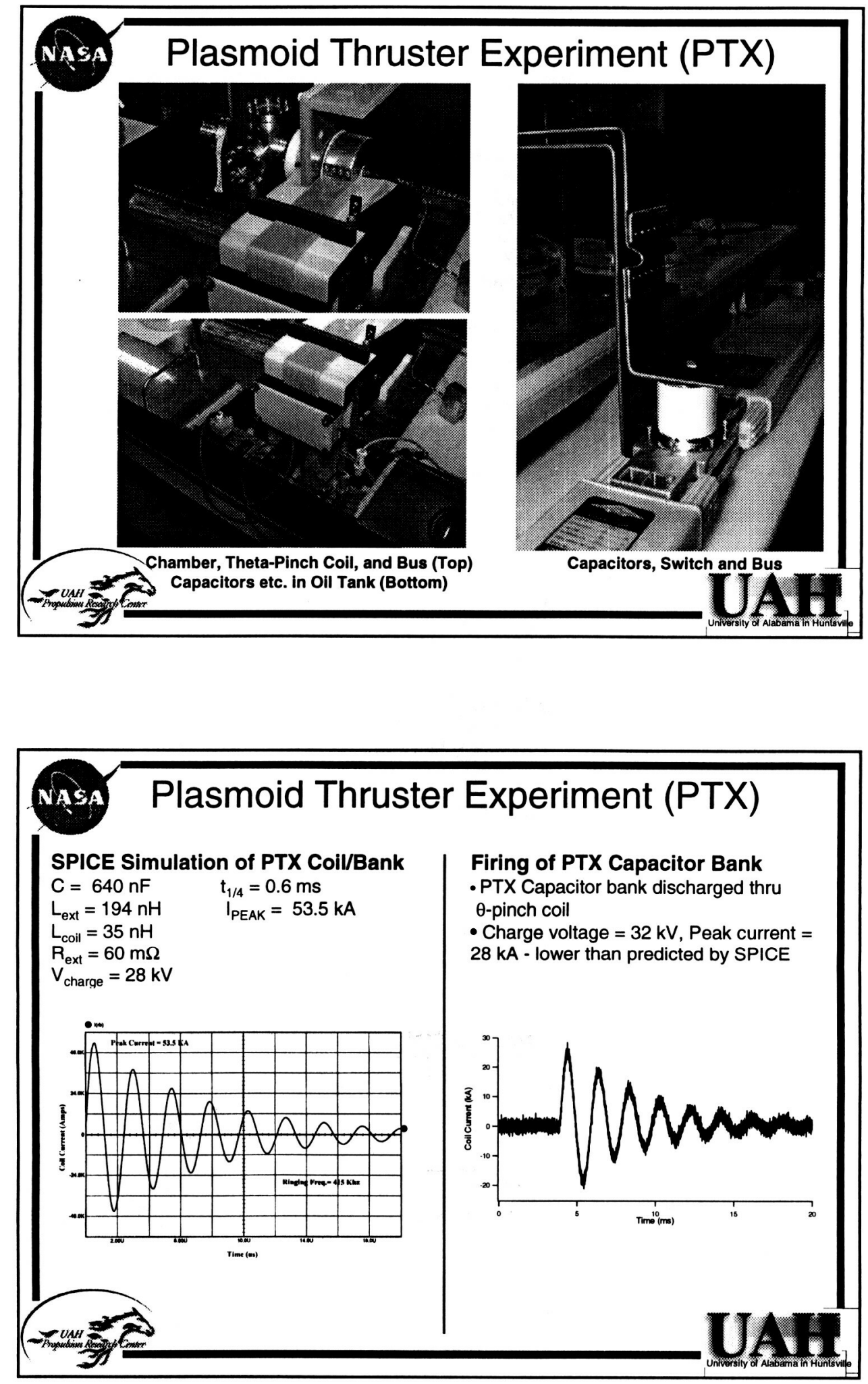

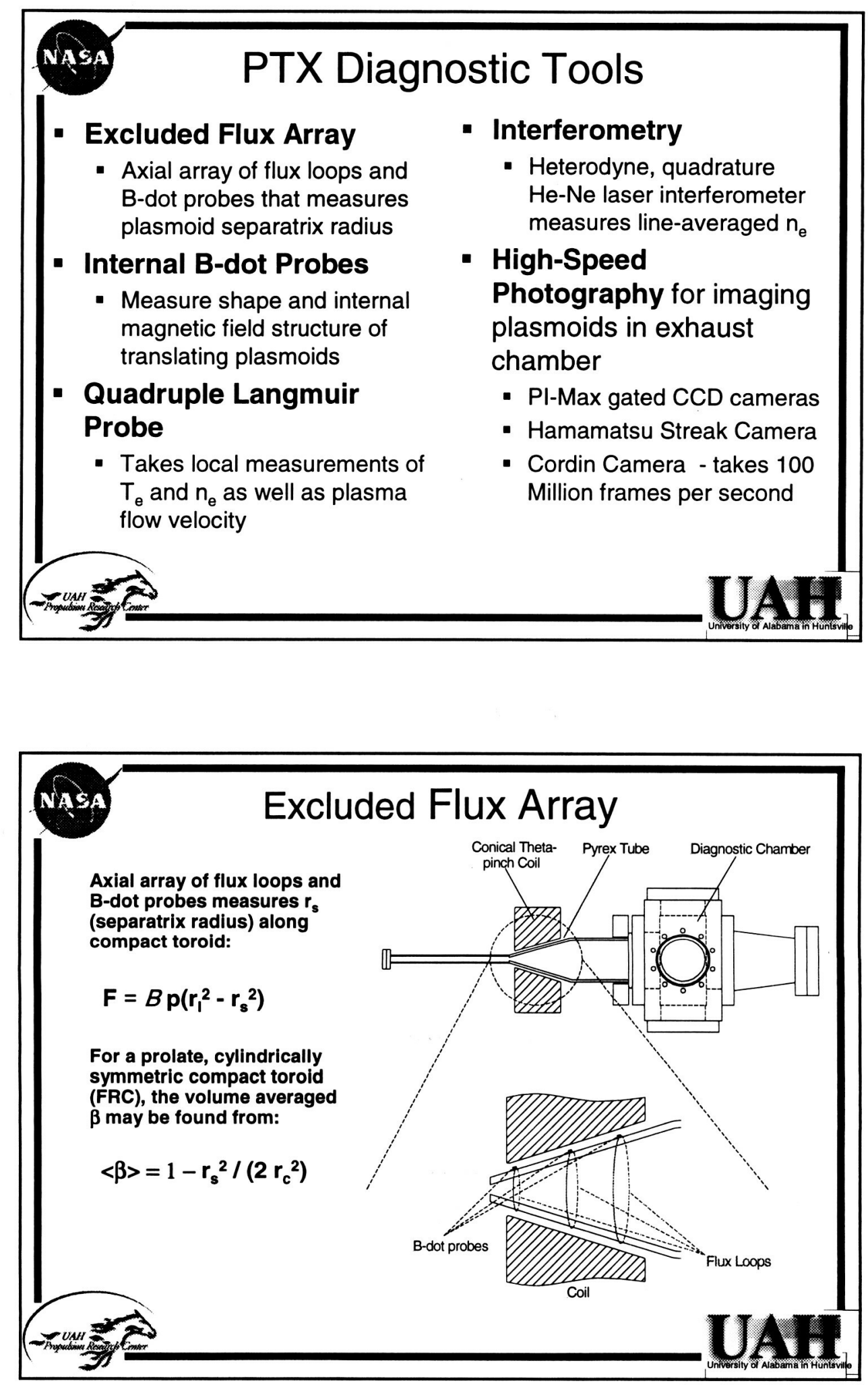

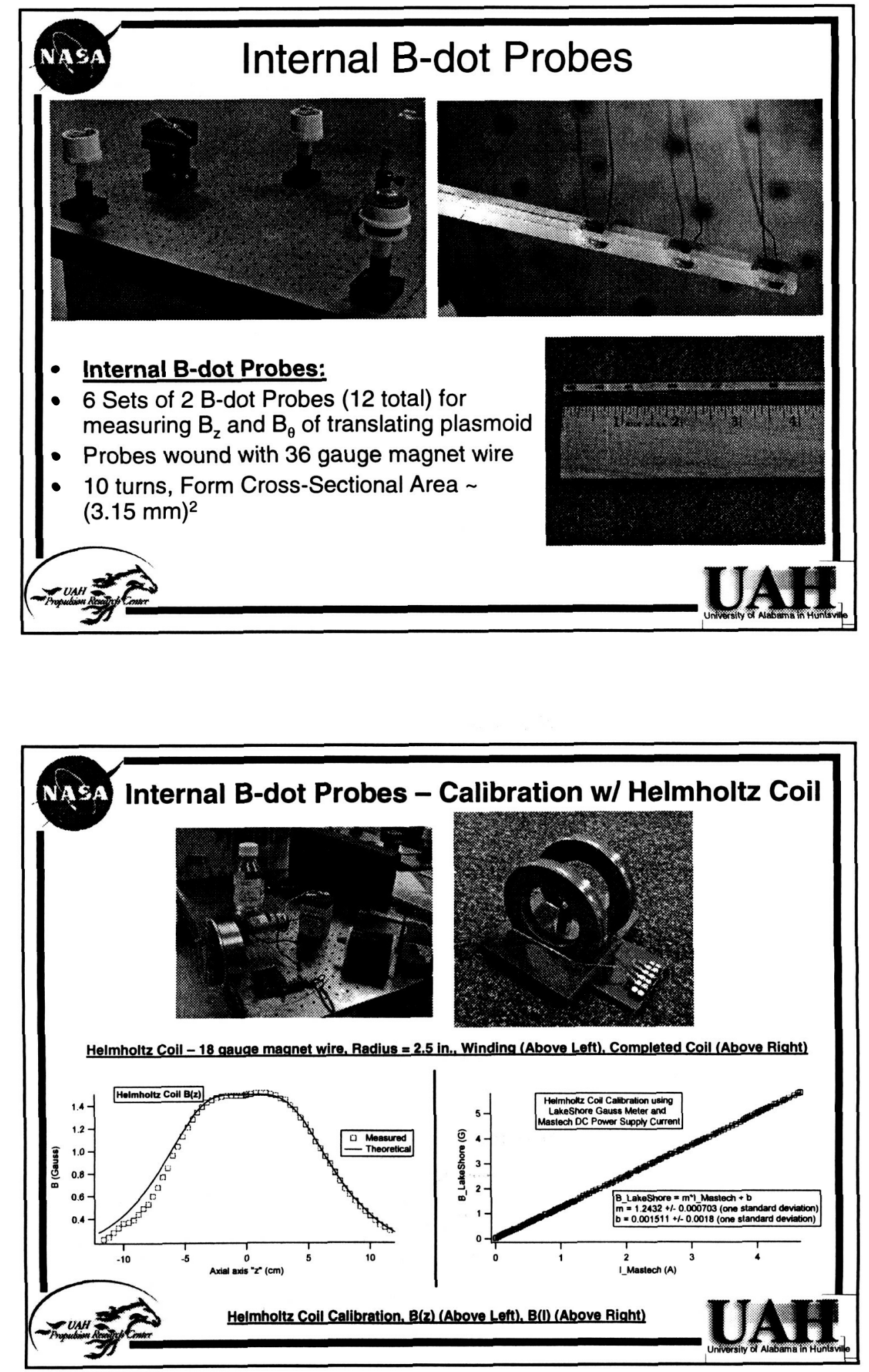

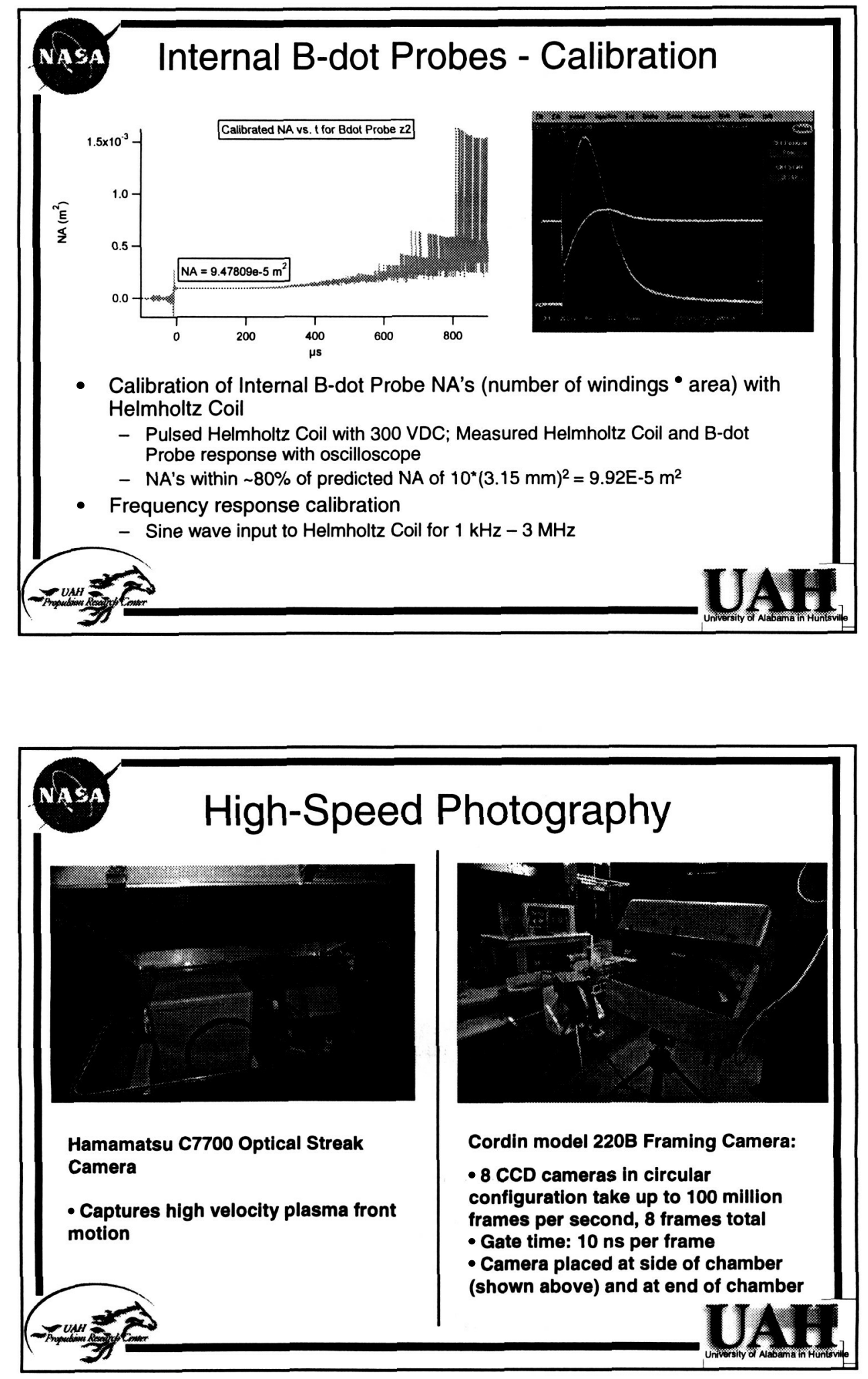


\section{Translating Plasmoid}

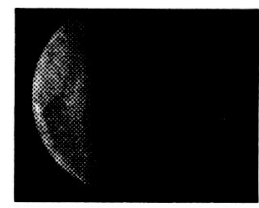

21 us

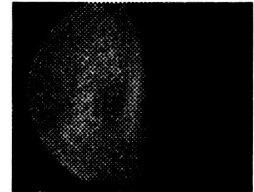

25 us

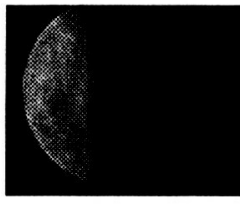

22 us

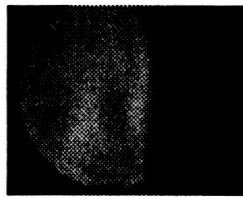

26 us

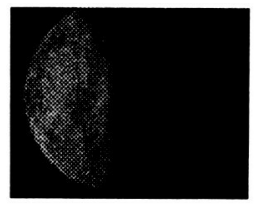

23 us

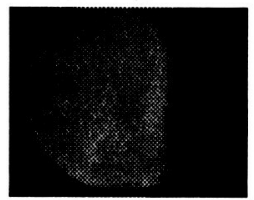

27 us

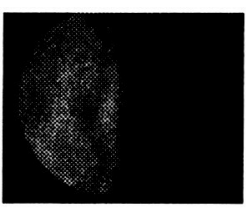

24 us

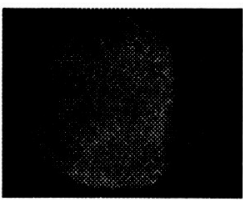

28 us

Propagating plasmoid in Air at $43 \mathrm{mTorr}$ backfill pressure (view from side of vacuum chamber); Velocity for this test was $5.3 \mathrm{~km} / \mathrm{s}$
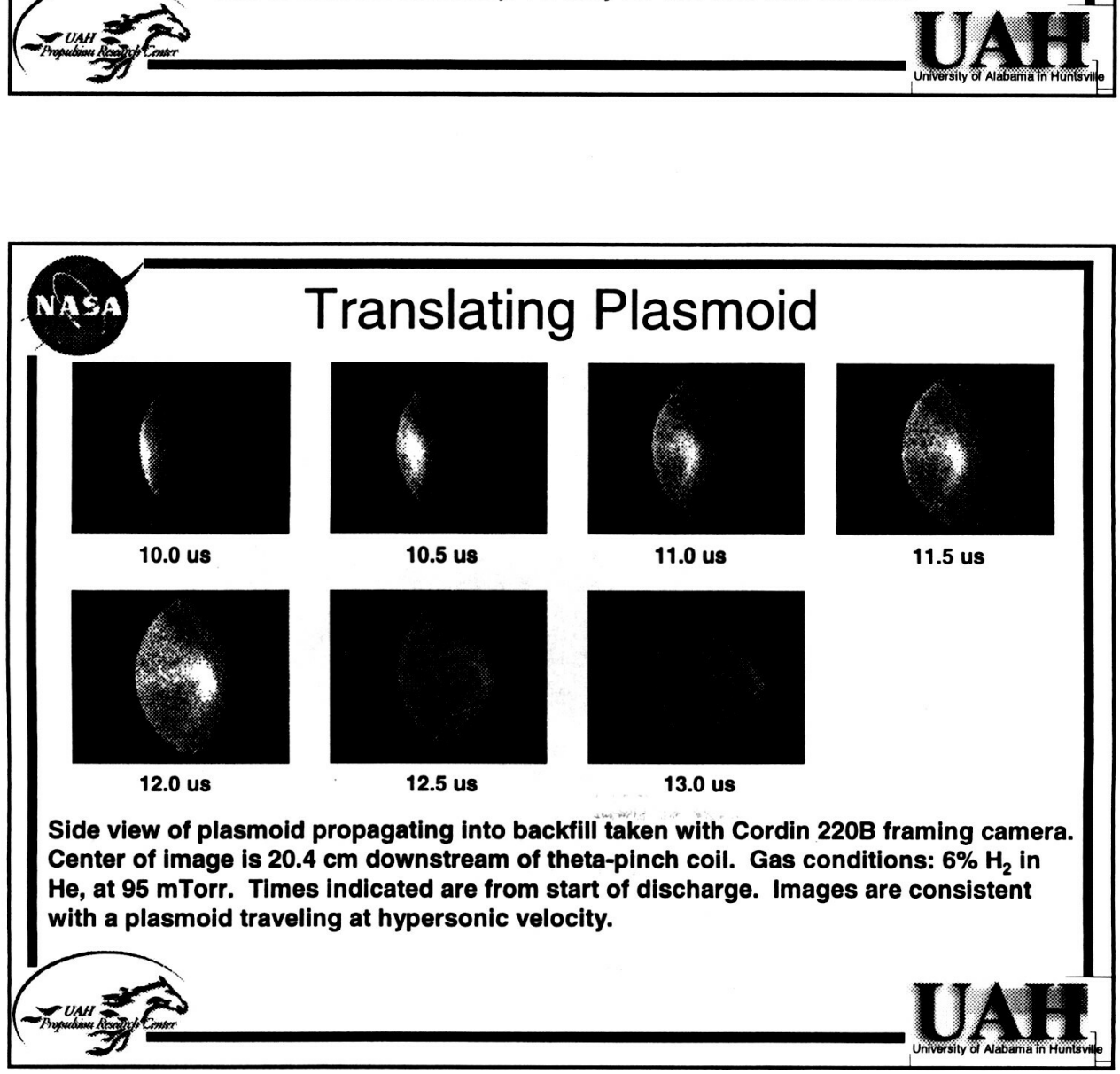

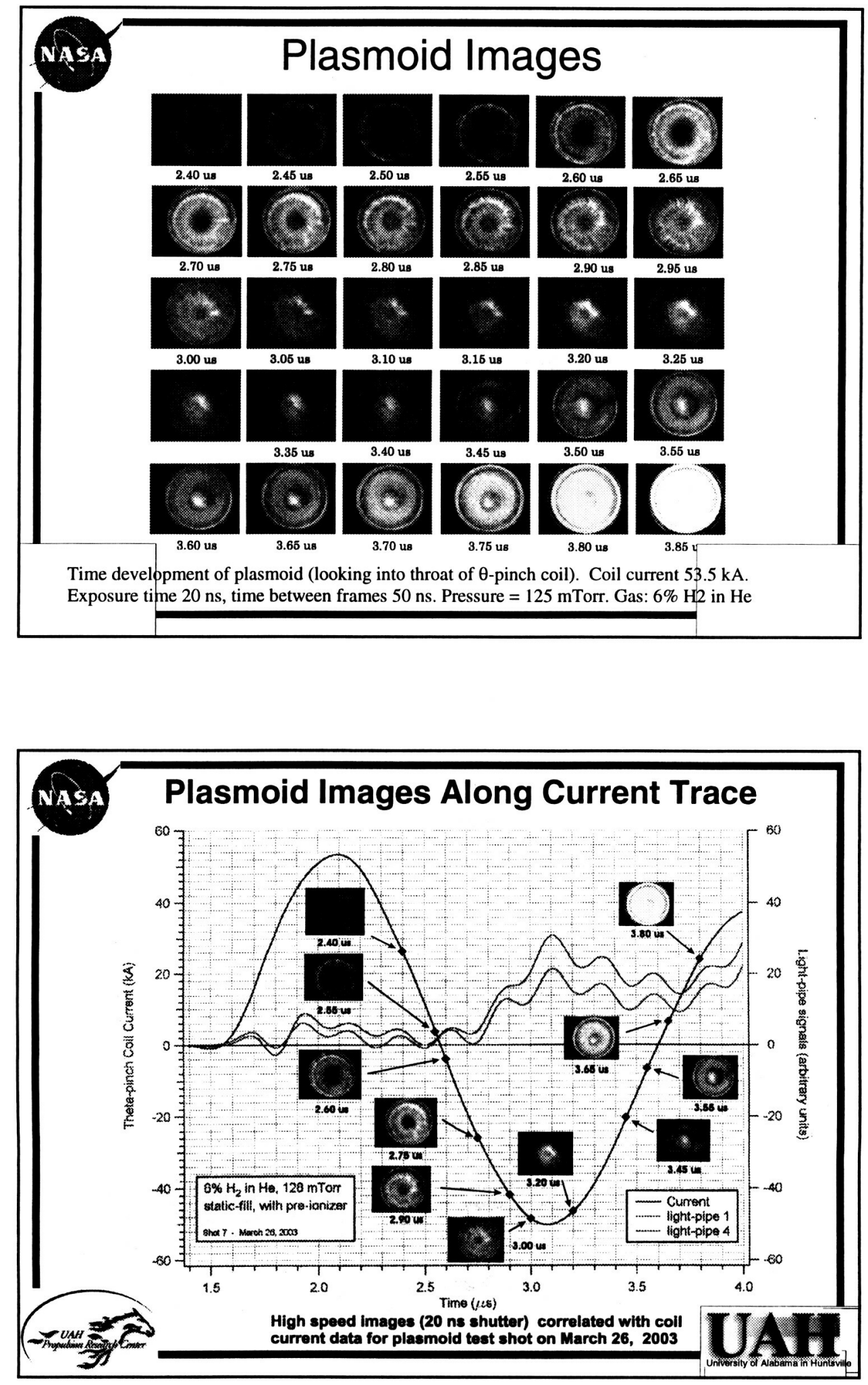


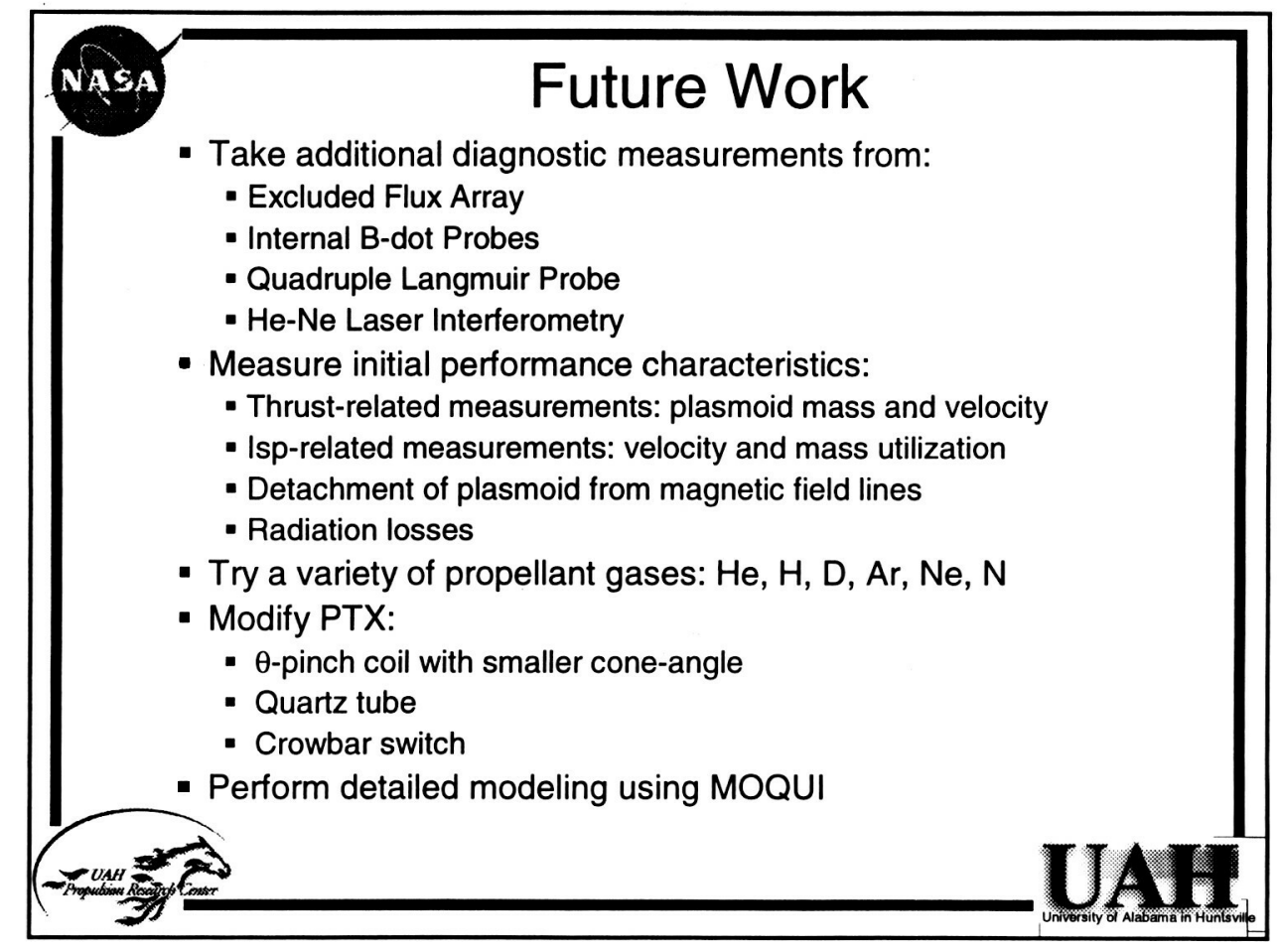

